\title{
Torsion of the uterus with myomas in a postmenopausal woman - case study and review of the literature
}

\author{
Dobrosława Sikora-Szczęśniak ${ }^{1}$, Grzegorz Szczęśniak², Tomasz Łęgowik², Wacław Sikora ${ }^{1}$
}

${ }^{1}$ Department of Obstetrics and Gynecology, Specialist Hospital in Radom

${ }^{2}$ Department of General Surgery, Specialist Hospital in Radom

${ }^{3}$ Department of Radiology, Specialist Hospital in Radom

\begin{abstract}
Introduction: Torsion of the uterus is defined as a rotation of more than $45^{\circ}$ around the long axis of the uterus; $2 / 3$ of cases are dextrorotations. The extent of rotation usually ranges from $45^{\circ}$ to $180^{\circ}$.

Objective: The purpose of the article was to present a case study of a postmenopausal woman with uterine torsion and myomas and to review the articles discussing the problem of rotated non-pregnant uterus.

Material and methods: The article analyses the course of an extremely uncommon pathology, i.e. uterine torsion in a 67-year-old patient. Laparotomy exposed the uterus with myomas and numerous hemorrhages, rotated by $180^{\circ}$ to the right side, size of $350 \times 300 \times 200 \mathrm{~mm}$ and bilateral necrosis of the ovaries. Moreover, we present a review of articles discussing surgical management in case of rotated non-pregnant uterus.

Results and discussion: The patient was operated on by a team of gynecologists and surgeons. The uterus was derotated and total hysterectomy with salpingoophorectomy was performed. A fragment of the hepatic oval ligament was excised and periumbilical hernioplasty was performed. The patient was released home on the $10^{\text {th }}$ day following the operation.

Conclusions: If women complain of pain located within the small pelvis and abdominal cavity it is necessary to remember that it might result from the torsion of reproductive organs which is an uncommon condition but poses a health or life threat to patients. Surgical treatment of uterine torsion is successful if promptly implemented; in certain cases it is even possible to spare the patient's fertility.
\end{abstract}

Key words: uterine torsion, uterine myomas, surgical treatment.

\section{Introduction}

Torsion of the nonpregnant uterus is extremely rare [1-4]. The cases described in the compiled literature occurred in the peri- and postmenopausal period. They are even more infrequent in childhood [5, 6]. They produce such symptoms as abdominal pain, acute or chronic, that persists for several months, vaginal bleeding, gastro-intestinal disorders, and urinary tract problems including acute urine retention [1].

Diagnostic imaging methods, e.g. CT and MRI, are very useful to establish the pre-operative diagnosis $[4,7]$. When repeated USG scans reveal altered location of myomas it can direct suspected abnormality towards uterine torsion [8].

Neither the mechanism nor the etiology of uterine torsion has been determined [9]. Rotation of the nonpregnant uterus is related to adhesions within the small pelvis, uterine myomas or other uterine abnormalities [10]. Other causes include congenital or structural (after Caesarean section) anomalies between the uterine body and cervix, long or stiff cervix, giant ovarian cysts, and mitotic dystrophy - a type of Duchenne muscular dystrophy $[1,5,11]$.

\section{Aim of the study}

The purpose of the article was to present a case study of a postmenopausal woman with uterine torsion and myomas and to review the articles discussing the problem of rotated non-pregnant uterus.

\section{Material and methods}

The article analyses the course of a very rare pathology, i.e. torsion of the uterus with myomas in a 67-yearold patient. The material for analysis was the patient's medical records, the operation report and histopathological reports. The case study considered compiled articles on the subject published over the last several years. 


\section{Case study}

The patient reported to hospital on January $8^{\text {th }}, 2013$ for intermittent abdominal pain persisting for two days. The patient was diagnosed and necessary laboratory investigations and imaging scans of the abdominal and chest organs were performed.

Medical history findings: menarche - at the age of 15 years, menstruation every 35 days lasting for 3-4 days, moderate and painless. Marital status - single. No deliveries or miscarriages. Menopause - at the age of 58 years. On admission she reported slight bleeding from the uterus.

On examination: The patient was in a general good condition, but complained of abdominal pain. BP $130 / 100 \mathrm{~mm} \mathrm{Hg}$, pulse $80 / \mathrm{min}$, temperature $36.8^{\circ} \mathrm{C}$. Body weight $62 \mathrm{~kg}, \mathrm{BMI}=23.1 \mathrm{~kg} / \mathrm{m}^{2}$. Peritoneal signs negative.

Gynecological examination: A small amount of bloody contents present in the vagina on colposcopy. Initial examination: difficulty exposing vaginal part of the cervix on palpation and colposcopy, tenderness of the upper part of the vagina on palpation and colposcopy. Bimanual palpation: The whole body of the uterus enlarged, its fundus reaching the rib arches.

Laboratory results (January 10 $0^{\text {th }}, 2013$ ): Significant are: blood count; high $-\mathrm{WBC}-13.1 \times 10^{3} / \mathrm{mL}$ (reference

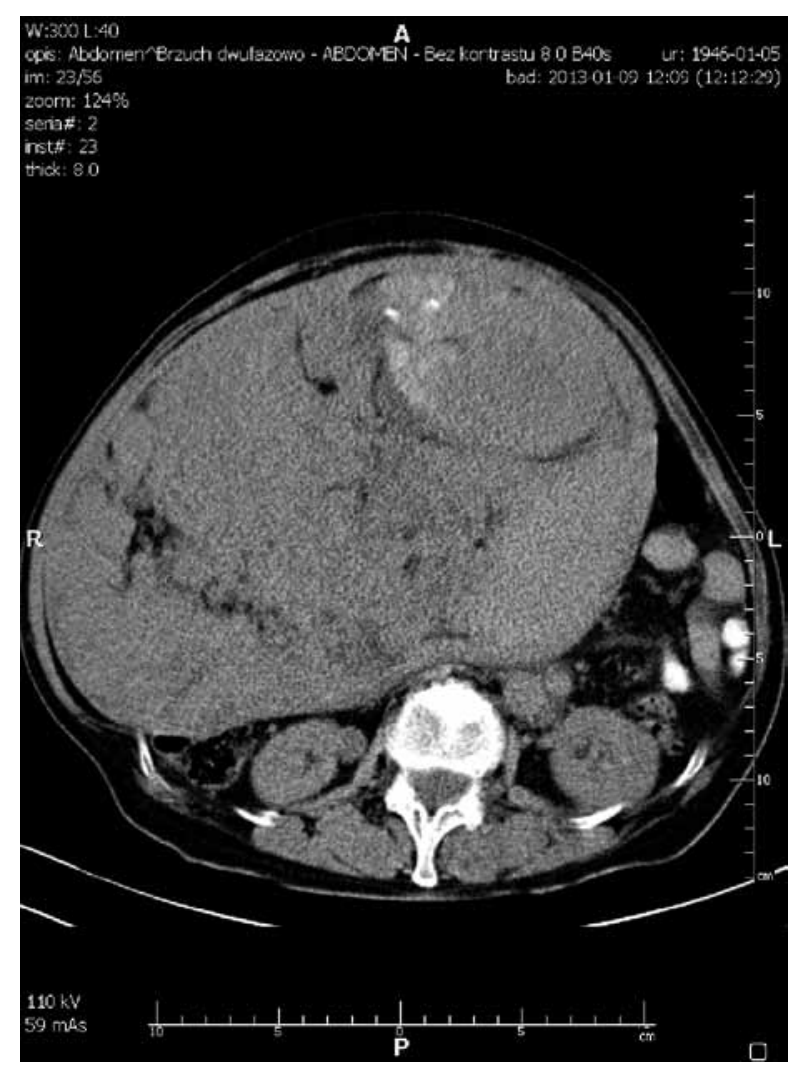

Fig. 1. Phase without dye. Visible hemorrhagic changes range 4-10), low - PLT - $84 \times 10^{3} / \mathrm{mL}$ (reference range 150-400).

Coagulogical test: PTT - high, INR - 1.31 (reference range 0.8-1.2), APTT - $32.6 \mathrm{~s}$ (reference range 25-45), low level of fibrinogen $-0.95 \mathrm{~g} / \mathrm{L}$ (reference range 2.04.0), high level of D-dimer $->20000 \mathrm{ng} / \mathrm{ml}$ (reference range < 500), antithrombin III - 75\% (norm 80-120). Tumor markers were high: CA-125 - $93.63 \mathrm{U} / \mathrm{mL}$ (reference range < 35.0), CA 19-9 - $77.4 \mathrm{U} / \mathrm{mL}$ (reference range 0.0-39.0).

USG scan taken on January $8^{\text {th }}, 2013$ : The whole abdominal cavity filled with a solid tumor of non-homogeneous echostructure.

Computed tomography of the abdominal cavity and small pelvis taken on January $9^{\text {th }}, 2013$. Cholecystolithiasis. Liquid present in the small pelvis. Tumor-like mass, size $272 \times 184 \times 240 \mathrm{~mm}$ of mixed density, not pronounced, with calcifications and fatty components visible, of smooth contour and considerable mass effect. The tumor is likely to press the right ureter and inferior vena cava, displacing intestinal loops. Tumor-like mass of slightly non-homogeneously increased density - areas of post-hemorrhagic origin should be considered within the tumor mass (Fig. 1). Visible wide vascular structures located peripherally. Possible rotation of the adnexa caused by tumor. Varicoid widening of the left ovarian vein (Fig. 2). Normal looking reproductive

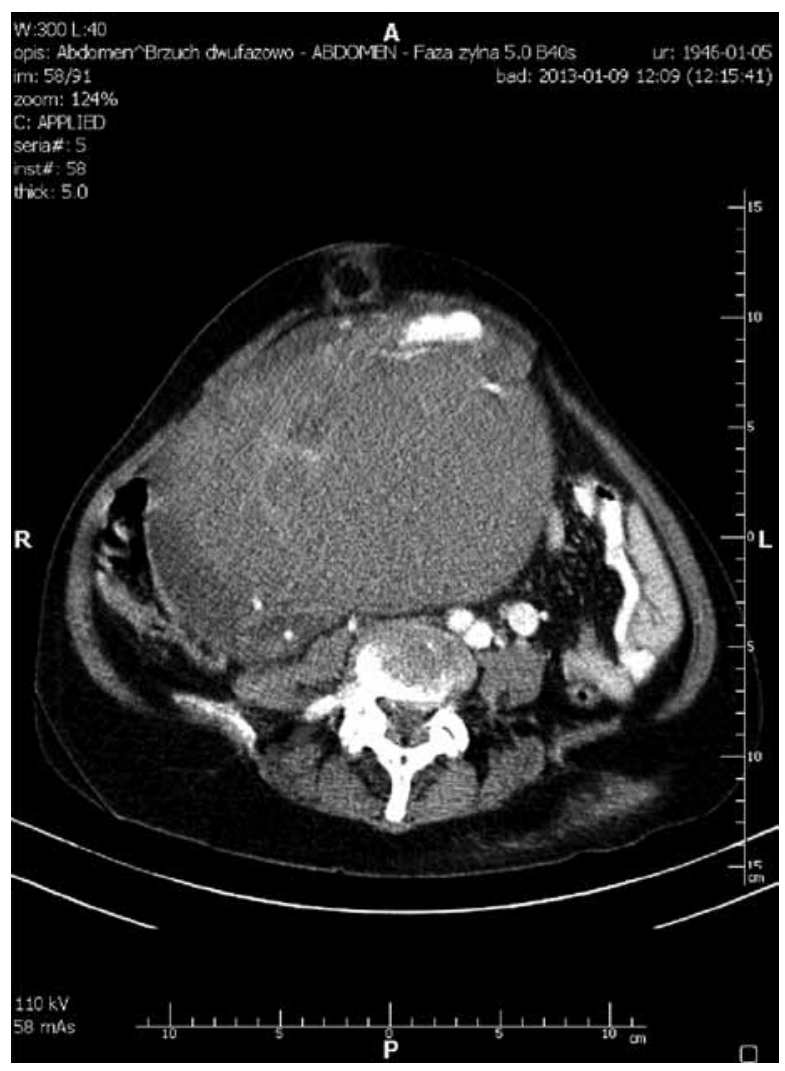

Fig. 2. Venous phase - wide veins visible on the left side. Configuration of the upper part of the vagina: X-shaped, vaginal veins widened 


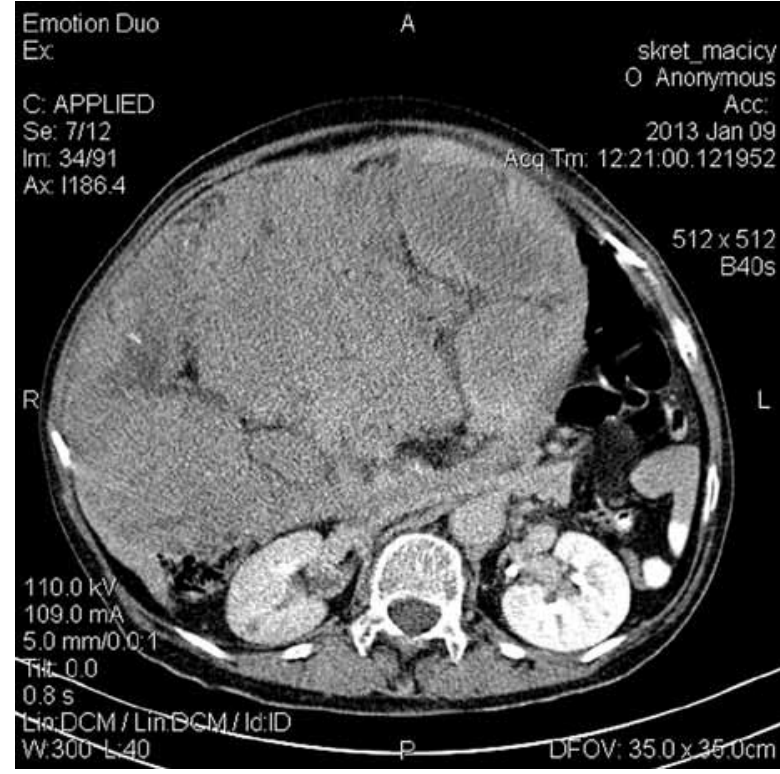

Fig. 3. Oppressed vessels of the right kidney

organs were not visualized. The right kidney less pronounced, oppressed (Fig. 3). Abdominal lymph nodes not enlarged. Periumbilical hernia.

The patient was consulted by an internist, surgeon and cardiologist and qualified for surgery which was performed by a team of gynecologists and surgeons (on January $\left.11^{\text {th }}, 2013\right)$.

Laparotomy: The whole of the uterine body was enlarged, of soft consistency, $350 \times 300 \times 200 \mathrm{~mm}$ in size,

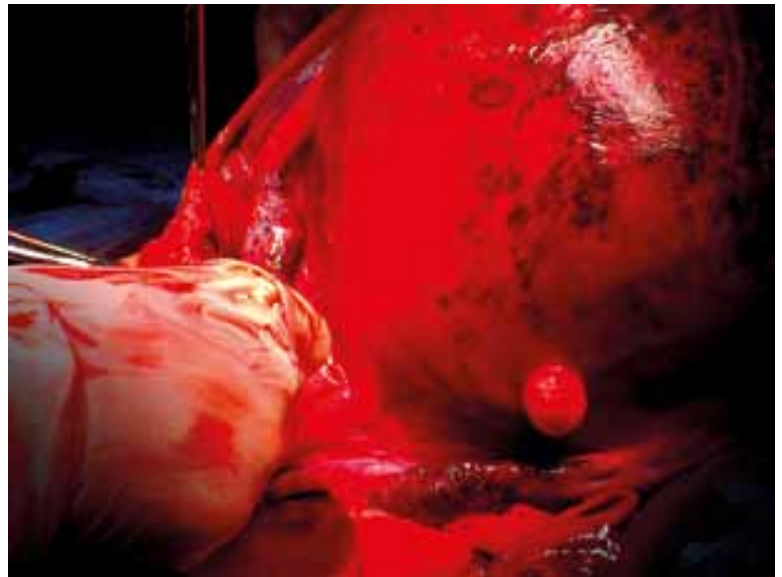

Fig. 4. Bilateral adnexa located anteriorly to the rotated uterus. Areas of hemorrhages into the uterus and ovaries

with bloody effusions on the surface. The body was dextrorotated by $180^{\circ}$. The adnexa were located anteriorly to the rotated uterus on both sides, swollen with intraovarian hemorrhages (Fig. 4).

The uterus was derotated, conventional total hysterectomy with bilateral salpingoophorectomy and periumbilical hernioplasty performed (Figs. 5 and 6).

Histopathological results: (The Department of Pathomorphology, Specialist Hospital, Radom). The uterine body: Endometrium atrophicum. Leiomyoma intramurale necroticans. The uterine cervix: Cervicitis chronica et haemorrhagiae diffusae recens. Both ovaries and Fallopian tubes: Necrosis haemorrhagica recens. Fragment

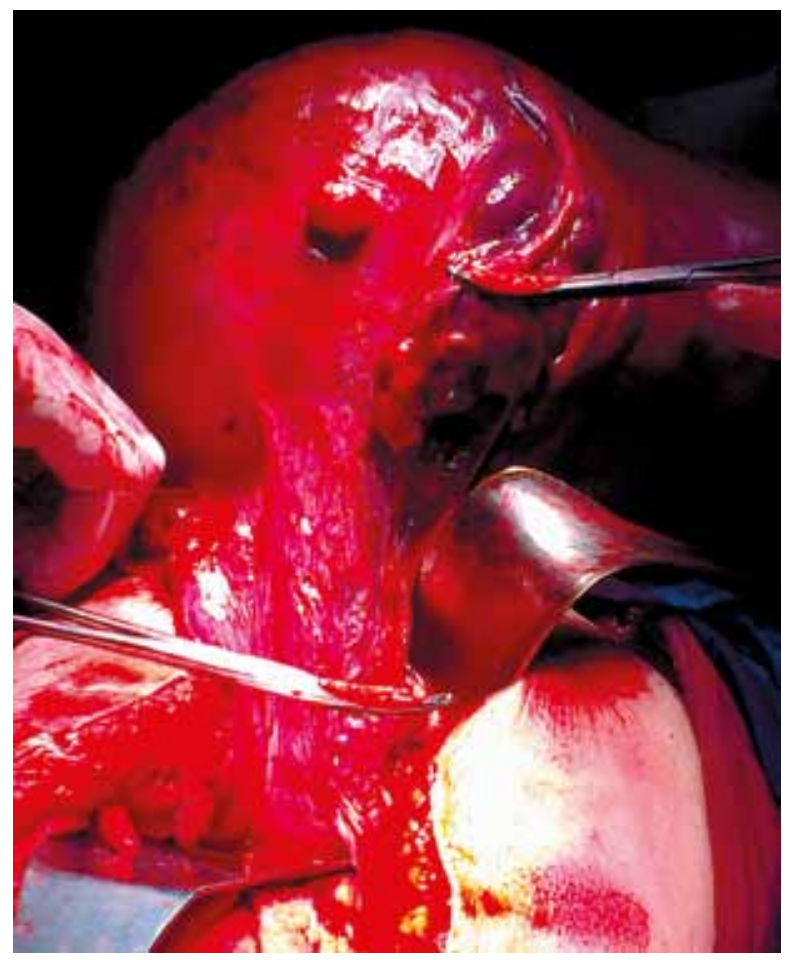

Fig. 5. Dissection of the uterus

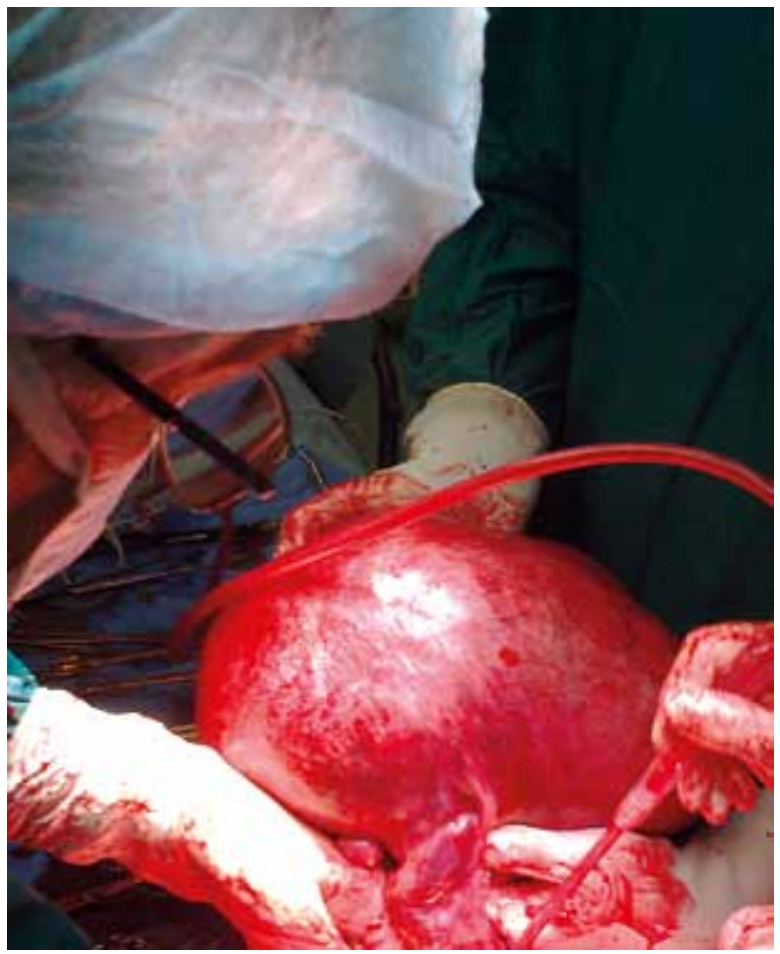

Fig. 6 . The uterus was derotated, total hysterectomy was performed 
of the greater omentum: Tela adiposa omenti. Hernial sac: Cutis et tele subcutaneae.

On the first day following the surgery the patient received $3 \mathrm{U}$ of packed red blood cells (PRBCs). The early post-operative course was uneventful. The patient was discharged from hospital on the $10^{\text {th }}$ day after the surgery.

\section{Discussion}

Torsion of the uterus is suspected infrequently during the pre-operative diagnostic process [11]. The final diagnosis of uterine torsion can be made only when laparotomy has been performed [12].

Typically the following signs and symptoms are found pre-operatively: abdominal pain, moderate bleeding from the uterus, rotation of the upper part of the vagina which produces difficulty inserting a vaginal speculum to expose the vaginal part of the cervix and initial signs of coagulopathy due to wear. Difficulty inserting the speculum to have a deeper insight into the cervix was not related to the rotation of the upper sectors of the vagina, associated with the rotation of the uterine body.

The compiled articles have noted various duration of the ailments in case of uterine torsion: 1-2 months until hospitalization and surgery $[3,12,13]$. It depended on the intensity of pain produced by a fast-rotating uterus and the degree of its rotation.

In the case of uterine torsion presented above, moderate intensity of the symptoms is a frequent manifestation in peri- and postmenopausal patients [12, 13].

Coagulogical tests revealed evidence of coagulopathy due to wear.

The symptoms of coagulopathy resulted from necrotic and hemorrhagic changes that usually develop when myomas are present and the adnexa have rotated $[3,5,6,10]$. Handler et al. presented coagulopathy caused by advanced necrotic angioleioma in the uterus [14]. Such degenerations result from ischemia and degenerative changes depending on the degree and rate of vascular failure [14].

When uterine torsion is detected, the decision to implement surgery is of key importance for successful treatment as it allows one to avoid the risk of developing ischemic, thrombotic and embolic complications which themselves make operative procedures even more complicated $[2,7,8]$.

Irreversible damage to the uterus due to ischemia can worsen the patient's clinical condition within a short time and pose a serious threat to life. In young girls and women in reproductive age a sparing operation undertaken at due time can maintain their ability to conceive in future $[5,6,13]$.

However, the above-mentioned complications resulting in massive bleeding, threatening the patient's health and life are an indication for hysterectomy, also in the case of Caesarean section undertaken for torsion of the gravid uterus [15-17].

Reading the CT scan of the abdominal cavity and small pelvis noted a characteristic suspicion of posthemorrhagic areas within the tumor and visualized wide vascular structures peripherally located. The results suggested possible torsion of the adnexa which was related to the tumor and varicoid enlargement of the left ovarian vein.

Computed tomography results were confirmed by histopathological tests. Detailed evaluation of the problem is possible on MRI [7, 8].

In this case, the upper part of the vagina was X-shaped, contrary to its normal $\mathrm{H}$-shape. The picture of the cervix visualized on the CT scan was more pronounced due to more difficult outflow of venous blood from the area [4].

However, access to advanced radiological imaging techniques such as CT or MRI is not always possible considering various circumstances [3].

Only after laparotomy has been done is it possible to find adnexa located anteriorly to the uterus due to its rotation.

It has to be emphasized that in the above case the torsion of the uterus could have been suspected preoperatively due to the complex clinical picture of the disease. Since the problem is extremely uncommon it is not always considered among other indications for surgical treatment, which is usually enforced by the patient's clinical state and implemented for life-saving reasons. That was also true in the case presented above.

Prompt surgical treatment undertaken in due course allows one to minimize the probability of developing sepsis (related to necrosis) and hemorrhage and lets one undertake surgery whose range has been planned. In women at reproductive age surgical procedures remove anatomical causes of torsion (adhesions, myomas, and ovarian cysts) and derotate the uterus to its anatomical position [5-7, 13]. In peri- and postmenopausal women total hysterectomy with salpingoophorectomy is performed.

In that case myoma may have affected the torsion of the uterus. The majority of reviewed articles presenting that problem noted myomas located within the uterine body, but large ovarian cysts were rare [4-6, 11].

The prophylaxis of thrombotic and embolic complications over the pre- and postoperative period followed the Protocol for Low Molecular Weight Heparin approved by the Team of Experts, Polish Gynecological Society [18].

\section{Conclusions}

1. If women complain of pain located within the small pelvis and abdominal cavity, it is necessary to re- 
member that it might result from the torsion of reproductive organs, which is a rare condition but poses a health or life threat to patients.

2. Surgical treatment of uterine torsion is successful if promptly implemented. In certain cases it is even possible to spare the patient's fertility or ovarian function.

\section{Disclosure}

The authors report no conflict of interest.

\section{References}

1. Varras M, Polyzos D, Alexopoulos Ch, et al. Torsion of a non-gravid leiomyomatous uterus in a patient with myotonic dystrophy complaining of acute urinary retention: anaesthetic management for total abdominal hysterectomy. Clin Exp Obstet Gynecol 2003; 30: 147-150.

2. Harada N, Haruta N, Nobuhara I, et al. A case of uterine torsion with a huge calciferous uterine leiomyoma in a postmenopausal (non-gravid) woman. Adv Obst Gynecol 2007; 59: 6-9.

3. Rajapaksha RKLS, Saklalasooriya PTB. A case of axial torsion of uterus with a large haemorrhagic leiomyoma. Sri Lanka J Obst Gynaecol 2011 33: 112-113.

4. Matsumoto H, Ohta T, Nakahara K, et al. Torsion of a nongravid uterus with a large ovarian cyst: usefulness of contrast MR image. Gynecol Obstet Invest 2007; 63: 163-165.

5. Dutra RA, Perez-Bóscollo AC, Ribeiro FC, et al. A laparoscopic approach to treating torsion of a nonpregnant uterus accompanied by a large ovarian cyst in a premenarchal girl. J Pediatr Surg 2008; 43: 17-19.
6. Grover S, Sharma Y, Mittal S. Uterine torsion: a missed diagnosis in young girls? J Pediatr Adolesc Gynecol 2009; 22: e5-8.

7. Luk SY, Leung JLY, Cheung ML, et al. Torsion of a nongravid myomatous uterus: radiological features and literature review. Hong Kong Med J 2010; 16: 304-306.

8. Nicholson WK, Coulson CC, McCoy MC, et al. Pelvic magnetic resonance imaging in the evaluation of uterine torsion. Obstet Gynecol 1995; 85 : 888-890.

9. Deshpande G, Kaul R, Manjuladevi P. A case of torsion of gravid uterus caused by leiomyoma. Case Rep Obstet Gynecol 2011; 2011: 206418.

10. Byun JM, Jeong DH, Lee YS, et al. Torsion of the uterus with leiomyoma in postmenopausal woman. Korean J Obstet Gynecol 2008; 51: 11871191.

11. Collinet P, Narducci F, Stien L. Torsion of a nongravid uterus: an unexpected complication of an ovarian cyst. Eur J Obstet Gynecol Reprod Biol 2001; 98: 256-257

12. Sankareswari R. Torsion of non-pregnant uterus due to fibroid alone is extremely rare and hence this case reported for its rarity. J Evol Med Dent Sci 2013; 2: 682-685.

13. Saquib S, Mathew M, Jain R. Torsion of a non-gravid uterus with leiomyoma mimicking broad ligament leiomyoma. Saudi Med J 2009; 30: 851-852.

14. Agarwal S, Gupta SK, Tejwani N. Angioleiomyoma of broad ligament. J Gynec Endosc Surg 2009; 1: 116-117.

15. Rasquinha SD, Rao SB, Rasquinha VC, et al. A twist in the tale - rare case of uterine torsion. Inter J Biomed Res 2012; 3: 434-436.

16. Thubert T, Razak RA, Villefranque V, et al. Uterine torsion in twin pregnancy. J Gynecol Obstet Biol Reprod (Paris) 2011; 40: 371-374.

17. Hariharan C, Shrivastava D, Walia M, et al. Torsion of gravid uterus near term - a rare complication. J Obstet Gynecol India 2009; 59: 455-456.

18. Low-wieght heparin treatment in obstetrics and gynecology - the Polish Gynecological Society. Ginekol Pol 2010; 81: 311-314. 